\title{
CONSTRUÇÃO E VALIDAÇÃO DE CONTEÚDO DO PLANO MULTIPROFISSIONAL DE ALTA EM TERAPIA INTENSIVA
}

\section{Thatiana Lameira Maciel Amaral}

Docente do Mestrado de Saúde Coletiva e da Residência multiprofissional Hospitalar com ênfase em Terapia Intensiva da Universidade Federal do Acre (UFAC) do Centro de Ciências da Saúde e do Desporto, Rio Branco (AC) Brasil.

\section{Kelly Regina Pires da Silva Caciano}

Enfermeira, residente do Programa de Residência multiprofissional Hospitalar com ênfase em Terapia Intensiva da Universidade Federal do Acre (UFAC) do Centro de Ciências da Saúde e do Desporto, Rio Branco (AC) Brasil.

\section{Bruna Martins de Carvalho}

Fisioterapeuta, residente do Programa de Residência multiprofissional Hospitalar com ênfase em Terapia Intensiva da Universidade Federal do Acre (UFAC) do Centro de Ciências da Saúde e do Desporto, Rio Branco (AC) Brasil.

\section{Jakeline de Lima Israel}

Enfermeira, residente do Programa de Residência multiprofissional Hospitalar com ênfase em Terapia Intensiva da Universidade Federal do Acre (UFAC) do Centro de Ciências da Saúde e do Desporto, Rio Branco (AC) Brasil.

\section{Irla Maiara Silva Medeiros}

Nutricionista, residente do Programa de Residência multiprofissional Hospitalar com ênfase em Terapia Intensiva da Universidade Federal do Acre (UFAC) do Centro de Ciências da Saúde e do Desporto, Rio Branco (AC) Brasil.

\section{Manuela Albuquerque Lima Ribeiro}

Fisioterapeuta, residente do Programa de Residência multiprofissional Hospitalar com ênfase em Terapia Intensiva da Universidade Federal do Acre (UFAC) do Centro de Ciências da Saúde e do Desporto, Rio Branco (AC) Brasil.

\section{Celso Gustavo Ritter}

Nutricionista, residente do Programa de Residência multiprofissional Hospitalar com ênfase em Terapia Intensiva da Universidade Federal do Acre (UFAC) do Centro de Ciências da Saúde e do Desporto, Rio Branco (AC) Brasil

Autor correspondente:

Thatiana Lameira Maciel Amaral

E-mail: thatianalameira27@gmail.com
RESUMO: O presente estudo objetiva construir e validar o conteúdo do instrumento de sistematização da continuidade do cuidado, mediante plano multiprofissional de alta. Foram realizadas as seguintes etapas: levantamento bibliográfico nas bases de dados Pubmed e BVS; construção do instrumento; validação do conteúdo por 20 especialistas, segundo técnica Delphi, e análise e consolidação dos itens do instrumento, sendo calculado o índice de concordância para os itens linguagem, pertinência, relevância, organização, clareza e aplicabilidade. A concordância entre os avaliadores variou de 85,0 a 100,0\%, com uma concordância global de 94,2\% para o modelo inicial. Foram incluídas dez $(23,2 \%)$ variáveis e 31 itens $(72,0 \%)$ sofreram modificações. Após a validação do plano, o instrumento permaneceu com 11 domínios e 55 itens. Este estudo favoreceu o planejamento para uma alta eficaz, por meio do uso de instrumento validado, para a obtenção de uma assistência multiprofissional de qualidade.

PALAVRAS-CHAVE: Estudos de Validação; Unidade de terapia intensiva; Equipe de Assistência ao Paciente.

\section{CONSTRUCTION AND VALIDATION OF MULTI-PROFES- SIONAL DISCHARGE PLAN IN INTENSIVE THERAPY}

ABSTRACT: Construct and validate the contents of a tool for the systematization of care continuity by means of a multi-professional discharge plan. The following stages were taken: bibliographic survey of PubMed and BVS databases; construction of the tool; validation of contents by 20 specialized professionals, following Delphi's technique, analysis and consolidation of the tool's items by calculating the agreement index for language, pertinence, relevance, organization, clarity and applicability. Agreement among evaluators varied between 85.0 and $100.0 \%$, with an overall $94.2 \%$ for the first model. Ten variables (23.2\%) were included and 31 items (72.0\%) were modified. After the plan's validation, the tool remained with 11 domains and 55 items. Current study enhanced planning with great efficiency to obtain quality multi-professional assistance.

KEY WORDS: Validation Studies; Intensive Care Units; Patient Care Team.

\section{INTRODUÇÃO}

A Unidade de Terapia Intensiva (UTI) é um local dotado de grande tecnologia e condições para diversas intervenções de alta complexidade; onde atuam profissionais de várias áreas da saúde, prepara- 
dos para medidas de suporte avançado de vida a qualquer momento. O cuidado intensivo é muito abrangente e contempla, entre outros, a monitorização contínua, atenção aos sinais e sintomas, avaliação diária e intervenções terapêuticas desempenhada de forma multiprofissional, objetivando uma assistência que permita, ao paciente, a alta em tempo e condições adequados ${ }^{1}$.

Por vezes, a alta da unidade de terapia intensiva acaba por expor os pacientes a diferentes níveis de cuidado, o que pode resultar em mortes inesperadas ou readmissões. Seus efeitos são acentuados quando a alta ocorre de forma precoce, uma vez que a readmissão, no geral, proporciona piora ou agravamento do quadro inicial com aumento da morbimortalidade e do tempo de internação ${ }^{2}$.

A manutenção efetiva da assistência após a alta da UTI contribui com a adequada recuperação do paciente, redução dos custos no tratamento e de possíveis complicações. É necessária a participação da equipe multiprofissional qualificada para suprir todas as demandas desses indivíduos e de seus familiares, além de recursos, instrumentos de registro e direcionamento da assistência, para a continuidade do cuidado ${ }^{3}$.

A utilização do planejamento da alta da UTI é uma ferramenta importante para a integralidade, continuidade, condução e coordenação do cuidado, com atenção aos processos terapêuticos multidisciplinares nos demais setores hospitalares. Esse recurso valoriza e inclui a família no processo de cuidado, a fim de dar prosseguimento e qualidade ao atendimento na enfermaria ou domicílio $^{4,5}$.

Assim, a alta deve basear-se em um plano de cuidados, desenvolvendo instrumento individualizado de acordo com as necessidades de cada paciente, desenhado desde o momento da sua internação, e que permita a estreita comunicação entre os responsáveis pela promoção da assistência de forma humanizada e integral ${ }^{6,7}$.

A validação do conteúdo do instrumento julga com precisão o objetivo a ser estudado, por meio de um comitê de especialistas. Compreende uma etapa fundamental para o desenvolvimento e adequação à realidade dos setores, com sua posterior implementação ${ }^{8}$.

Diante do exposto, o presente estudo tem por objetivo construir e validar o conteúdo do instrumento de sistematização da continuidade do cuidado, mediante plano multiprofissional de alta desenvolvido para UTI adulto, visando promover a comunicação eficaz entre os profissionais dos diferentes setores da unidade hospitalar, e destes com os familiares, para a obtenção de uma assistência de qualidade voltada à redução das incapacidades e da morbimortalidade após a UTI.

\section{METODOLOGIA}

Trata-se de um estudo metodológico para construção e validação de conteúdo do instrumento para sistematização da continuidade do cuidado, mediante plano multiprofissional de alta da UTI adulto. O presente estudo foi desenvolvido pela equipe de residência multiprofissional em terapia intensiva, da Universidade Federal do Acre, realizado nas UTIs públicas de Rio Branco, Acre, em outubro de 2017.

A construção e validação de conteúdo do plano multiprofissional de alta seguiram as seguintes etapas: levantamento bibliográfico; construção do instrumento e das instruções de utilização; validação do conteúdo pelos juízes; análise e consolidação dos itens do instrumento de coleta de dados.

Na primeira etapa foi realizada uma revisão integrativa da literatura ${ }^{9,10}$, em que se contemplam as seguintes fases:

1) identificação do tema e seleção da questão de pesquisa; essa etapa inclui definição das palavras-chaves utilizadas, que foram: estudo de construção e validação, instrumento; unidade de terapia intensiva, e equipe multiprofissional, além da elaboração da questão de pesquisa: "Como construir e validar o conteúdo do plano multiprofissional de alta em terapia intensiva?"

2) estabelecimento de critérios para inclusão e exclusão dos estudos/ amostragem ou busca de literatura: a estratégia de busca foi realizada nas bases de dados PubMed e BVS, das publicações dos últimos dez anos. Como critérios de inclusão foram os artigos originais 
completos periódicos em português, inglês e espanhol que apresentassem no título ou resumo os seguintes termos de busca: estudos de validação, unidade de terapia intensiva, equipe multiprofissional, enfermagem, nutrição, fisioterapia e relatório de alta. Os critérios de exclusão foram referências de teses, dissertações e livros;

3) definição das informações a serem extraídas dos estudos selecionados/categorização dos estudos: as informações obtidas incluíram identificação do artigo (título, periódico, ano de publicação, ano de realização da pesquisa e palavras-chaves); identificação do(s) autor (es); e características do artigo científico (amostra, objetivos, método, resultados e conclusão). Para aprimorar a amostra foi realizada a leitura e fichamento independente dos resumos selecionados, observando os critérios acima mencionados;

4) avaliação dos estudos incluídos na revisão integrativa: a seleção foi realizada em duas fases: a) avaliação dos títulos e dos resumos de todos os estudos selecionados, de forma independente, por dois avaliadores; b) posterior à seleção dos resumos, os artigos foram analisados por completo criticamente, por três pesquisadores de forma independente;

5) interpretação dos resultados: mediante análise descritiva após leitura na íntegra dos artigos selecionados, foram construídas categorias analíticas para a discussão dos resultados;

6) apresentação da revisão/síntese do conhecimento: seguindo avaliação de acordo com a pertinência na elaboração da revisão, a inclusão de informações obtidas, com base no protocolo da pesquisa, foi sintetizada e descrita de forma analítica.

Posteriormente, mediante embasamento científico, conforme descrito acima, foi construído o instrumento com nove domínios, composto de 43 itens, com questões abertas e fechadas da sistematização da continuidade no cuidado após UTI nas áreas da enfermagem, fisioterapia e nutrição.
Os itens, de modo geral, contemplam dados pessoais do paciente, história pregressa, diagnósticos atuais e anteriores, procedimentos realizados (exames e intervenções), itens de semiologia, evolução e prescrições da enfermagem, da nutrição e da fisioterapia, cuidado psicossocial e orientações aos familiares, além de campo destinado à descrição dos enfoques do cuidado. As instruções para preenchimento e utilização do instrumento também foram criadas com intuito de facilitar o entendimento dos itens.

Na etapa de validação do conteúdo pelos especialistas foi utilizada a técnica Delphi, em três fases que incluem a seleção dos especialistas; apresentação do instrumento e orientação para a avaliação do conteúdo do roteiro quanto à aplicabilidade, relevância, pertinência e clareza, sendo ainda obtidas informações sobre linguagem e organização.

O grupo de especialistas foi composto por 20 profissionais entre enfermeiros $(35,0 \%)$, fisioterapeutas (35,0\%), nutricionistas $(25,0 \%)$ e psicólogos $(5,0 \%)$ que possuíam, no mínimo, dois anos de experiência na área de terapia intensiva na assistência ou docência, sendo estes pós-graduados (especialistas, mestres e doutores) na área de interesse ou ainda em áreas afins ao tema. Vale ressaltar que não houve a participação dos profissionais de medicina por estes possuírem instrumento próprio de relatório de alta.

Para proceder a avaliação, em fase exploratória, foi apresentado aos especialistas o instrumento e realizada as devidas instruções dos itens de avaliação. A análise do instrumento baseava-se no julgamento em adequado ou inadequado, sendo possível a sugestão de inclusão, exclusão ou modificação de itens. O instrumento foi aplicado para avaliação em local tranquilo e sem interrupções para garantir a qualidade da análise.

$\mathrm{Na}$ validação do conteúdo foi utilizada a medida do índice de concordância adotada para inclusão e/ ou exclusão e/ou modificações nos itens do instrumento, sendo aceito o valor igual ou superior a $80 \%$ entre os juízes ${ }^{11}$. Os itens que não obtiveram concordância, assim como os novos itens inseridos, foram analisados individualmente, quanto à pertinência pelos pesquisadores. $\mathrm{O}$ percentual de concordância é obtido pela divisão entre o número de participantes que concordaram pelo número 
total de participantes multiplicado por 100 . O julgamento dos especialistas foi tabulado em planilha Excel@, levando em consideração os comentários e sugestões.

A pesquisa atendeu às normas da Resolução do Conselho Nacional de Saúde 466/12, aprovada pelo Comitê de Ética em Pesquisa da Universidade Federal do Acre, com o parecer número 1.336.173.

\section{RESULTADOS}

A concordância entre os avaliadores variou de 85,0 a 100,0\% nos itens organização, adequação, clareza, linguagem, pertinência, continuidade e conteúdo (Tabela 1). Os itens de avaliação acima mencionados têm o mesmo peso. Quanto às variáveis, foram incluídas dez $(23,2 \%), 31$ itens $(72,0 \%)$ sofreram modificações e nenhum foi excluído.

Tabela 1. Concordância entre avaliadores do instrumento do plano multiprofissional de alta em terapia intensiva. Rio Branco, Acre, 2017

\begin{tabular}{lc}
\hline \multicolumn{1}{l}{ Itens de avaliação } & Concordância (\%) \\
\hline Linguagem & 100,0 \\
Pertinência & 100,0 \\
Relevância & 100,0 \\
Organização & 90,0 \\
Clareza & 90,0 \\
Aplicabilidade & 85,0 \\
\hline
\end{tabular}

A partir da análise das avaliações, realizadas pelos juízes, foi sugerida inclusão de itens como número do prontuário no campo inicial de identificação do(a) paciente. Os avaliadores apontaram a necessidade de definir o profissional responsável pelo preenchimento dos domínios referentes ao histórico e diagnóstico. Foi ressaltada, também, a importância quanto à inclusão do domínio "intercorrências" após os dados de internação na UTI, além das prescrições de enfermagem no campo de evolução multiprofissional.

Sobre as variáveis de nutrição, houve sugestão de inclusão do estado nutricional, peso estimado na alta, percentual de VET, quantidade de proteína prescrita na alta, consistência da dieta de via oral e posicionamento da sonda de alimentação. Acerca do domínio de fisioterapia, foi proposto contemplar a necessidade de ventilação mecânica na internação na UTI, presença de tosse eficaz, risco de broncoaspiração e controle de tronco e cabeça. Para complementar, foram mencionadas a inclusão do escore de Medical Researche Council (MRC) e a avaliação do tônus muscular. No item que retrata o atendimento psicossocial, em que é abordada a atuação do profissional de psicologia frente ao paciente e seus familiares, assim como a sinalização da necessidade de atendimento da assistência social.

Alguns itens foram modificados para melhor leitura, organização e objetividade no preenchimento dos dados. Tais modificações ocorreram nas intervenções e procedimentos, cuidados de enfermagem, uso de medicações, classificação do nível de consciência segundo escala de coma de Glasgow (ECG) e ajustes da alimentação. Segundo um dos avaliadores, três itens relacionados à quantidade, à cor e ao aspecto da secreção traqueal foram apontados para possível exclusão, ficando a critério dos pesquisadores a decisão.

Após a validação do plano multiprofissional de alta, o instrumento recebeu o acréscimo de dois seguimentos, passando a dispor de 11 domínios e 55 itens (aumento de 30,9\%). Os domínios da versão final foram: dados do paciente, história pregressa, internação, procedimentos, intercorrências, checklist de alta da unidade, evolução multiprofissional (enfermagem, fisioterapia e nutrição), atendimento psicossocial e metas do cuidado terapêutico (Figura 1). 


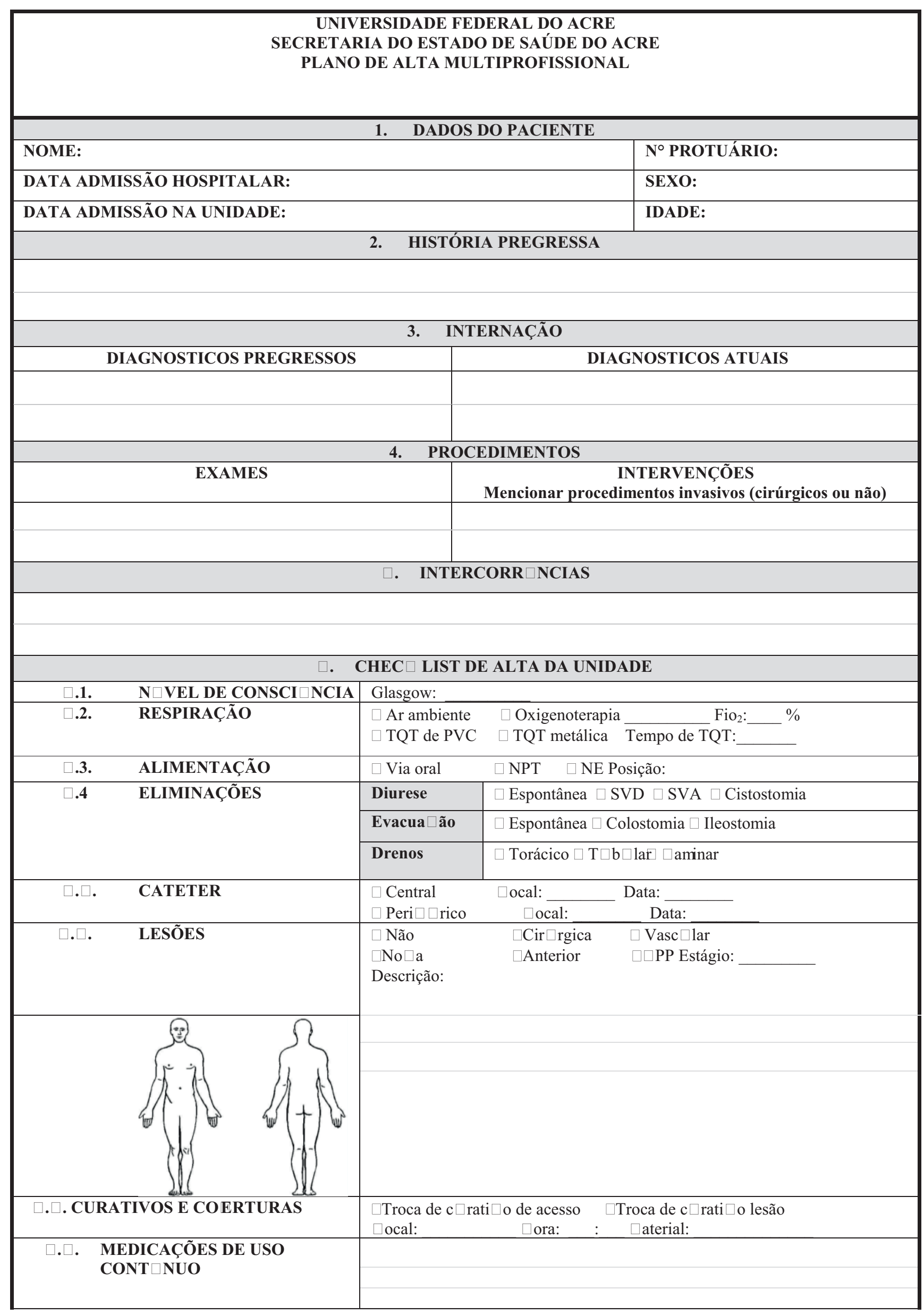




\begin{tabular}{|c|c|c|c|}
\hline \multicolumn{4}{|c|}{ EVOLUÇÃO MULTIPROFISSIONAL } \\
\hline \multicolumn{4}{|c|}{ 口. ENFERMAGEM } \\
\hline$\square \square \square \square$ onitorar $\mathrm{n} \square \square$ el de consci $\square$ ncia de $\square \square \square \square$ & \multicolumn{3}{|c|}{$\square \square \square \square$ eali $\square$ ar $\square$ idratação c $\square$ tânea ap $\square \mathrm{s} \mathrm{ban} \square$ o } \\
\hline $\begin{array}{l}\square \square 2 \square \square \text { eali } \square \text { ar troca de } \mathrm{c} \square \text { rati } \square \text { eom acesso } \square \text { enoso } \\
\text { obser } \square \text { ando sinais } \square \log \square \text { sticos } \square \text { dimenate o } \square \text { SN }\end{array}$ & \multicolumn{3}{|c|}{$\begin{array}{l}\square \square \square \text { eali } \square \text { ar balanço } \square \square \text { drico e registrar aspecto da di } \square \text { rese d } \\
\square \square \square \square\end{array}$} \\
\hline $\begin{array}{l}\square \square \square \text { Atentar para alteraç } \square \text { es } \square \text { emodinâmicas e } \square \square \text { eixas } \\
\text { álgicas }\end{array}$ & \multicolumn{3}{|c|}{$\begin{array}{l}\square \square \square \mathrm{A} \square \text { aliar FC } \square \mathrm{F} \square \square \mathrm{PAtmperat} \square \text { ra e per } \square \square \text { são } \\
\text { peri } \square \square \text { rica de } 2 \square 2\end{array}$} \\
\hline$\square \square \square \square$ eali $\square$ ar m danças de dec $\square$ bitøe $2 \square 2 \square$ & \multicolumn{3}{|c|}{$\square \square \square \square$ anter cabeceira ele $\square$ ada $\square \square \square$ a $\square \square$} \\
\hline \multicolumn{4}{|l|}{$\mathrm{O} \square$ tros: } \\
\hline \multicolumn{4}{|c|}{ NUTRIÇÃO } \\
\hline \multicolumn{4}{|c|}{ 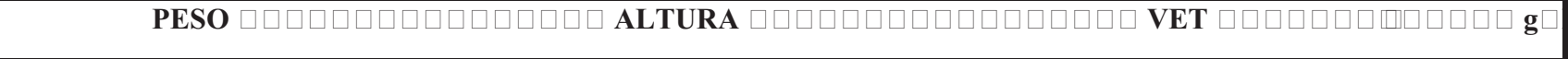 } \\
\hline \multicolumn{3}{|l|}{ Necessidade $\square \square$ drica } & \\
\hline \multicolumn{3}{|l|}{ Necessidades energ $\square$ ticas $\square$ VET $\square$ são atingidas $\square$} & $\square$ Sim $\square$ Não \\
\hline \multicolumn{3}{|l|}{ Necessidades proteicas são atingidas $\square$} & $\square$ Sim $\square$ Não \\
\hline \multicolumn{3}{|c|}{ Paciente com tend $\square$ ncia a desen $\square$ ol $\square$ er $\square \square$ adro diarreico $\square$} & $\square$ Sim $\square$ Não \\
\hline \multicolumn{3}{|c|}{ Paciente com tend $\square$ ncia a desen $\square$ ol $\square$ er $\square \square$ adronstipação $\square$} & $\square$ Sim $\square$ Não \\
\hline \multicolumn{3}{|c|}{ Degl $\square$ tição e $\square \square$ nção alimentar oral estão ade $\square \square$ adas $\square$} & $\square$ Sim $\square$ Não \\
\hline $\mathrm{O} \square$ erta cal $\square$ ricproteica na alta: & \multicolumn{2}{|l|}{ Estado $\mathrm{n} \square$ tricional: } & \\
\hline \multicolumn{4}{|c|}{ П. FISIOTERAPIA } \\
\hline Necessidade de VNI $\square$ & \multicolumn{2}{|l|}{$\square \operatorname{Sim} \quad \square$ Não } & \\
\hline$\square \mathrm{o} \square \square$ e a necessidade de $\square$ so o $\mathrm{V} \square \square$ & \multicolumn{2}{|l|}{$\square$ Sim $\square$ Não } & \\
\hline Necessidade de $\square$ igieni $\square$ ação br $\square \mathrm{n} \square \square$ ica $\square$ & \multicolumn{2}{|l|}{$\square$ Sim $\square$ Não } & \\
\hline$\square \square \square \square \quad$ Secreção tra $\square \square$ eal & $\square$ uantidade & $\square$ Grande $\square \square$ edia & $\square \mathrm{Po} \square \mathrm{ca}$ \\
\hline & Cor & $\begin{array}{l}\square \text { Amarelado } \\
\square \text { Es } \square \text { erdeado } \square \text { San } \\
\end{array}$ & $\begin{array}{l}\text { Esbran } \square \square \text { içado } \\
\text { g } \square \text { inolento }\end{array}$ \\
\hline & Aspecto & $\square \mathrm{F} 1 \square \square$ do $\square$ Espessa & $\square \square$ ol $\square$ osa \\
\hline Tosse e $\square$ ica $\square \square$ & $\square \operatorname{Sim} \square$ Não & & \\
\hline 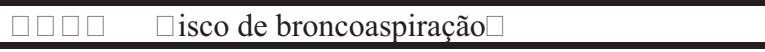 & $\square$ Sim $\square$ Não & & \\
\hline$\square \square \square \square \quad$ Alg $\square$ ma restrição de mobili $\square$ ação $\square$ & $\square \operatorname{Sim} \quad \square$ Não Q $\square$ al: & & \\
\hline$\square \square \square \square \quad \square$ isco de broncoaspiração $\square$ & $\square$ Sim $\square$ Não & & \\
\hline$\square \square \square \square \quad \square$ obili $\square$ ação artic $\square \operatorname{lar} \square$ & $\square$ Sim $\square$ Não & & \\
\hline$\square \square \square \square \quad \mathrm{A} \square \mathrm{x} \square$ lio para sedestação $\square$ ora do leito $\square$ & $\square$ Sim $\square$ Não & & \\
\hline$\square \square \square \square \quad \mathrm{A} \square \mathrm{x} \square$ lio para deamb $\square$ lação $\square$ & $\square$ Sim $\square$ Não & & \\
\hline Necessita de exerc $\square$ cio respirat $\square$ rio $\square$ & $\square$ Sim $\square$ Não & & \\
\hline$\square \square \square \square \quad$ Alteração de $\mathrm{t} \square \mathrm{n} \square \mathrm{s} \square$ & $\square$ Sim $\square$ Não Q $\square$ al: & & \\
\hline$\square \square \square \square \quad \mathrm{A} \square$ aliação do $\square \square \mathrm{C}$ & Escore: & & \\
\hline 1־. ATENDII & NTO PSICOSSOCIAL & & \\
\hline$\square \square \square \square \square$ Atendimento psicol $\square$ gico ao paciente & & & $\begin{array}{l}\square \text { Sim } \\
\square \text { Não }\end{array}$ \\
\hline$\square \square \square 2 \square \quad$ Necessidade de assist $\square$ ncia social $\square$ & & & $\begin{array}{l}\square \text { Sim } \\
\square \text { Não }\end{array}$ \\
\hline$\square \square \square \square \square$ Orienta $\square \square$ es $\square$ unto $\square$ m $\| \mathbf{a}$ ia $\square$ cuidador: $\square$ an & $\square \square$ edicaç $\square$ es & $\square$ Alimentação & $\square \mathrm{C} \square$ idados \\
\hline$\square \square$ anip $\square$ lação $\square \mathrm{O} \square$ tro & & & \\
\hline
\end{tabular}




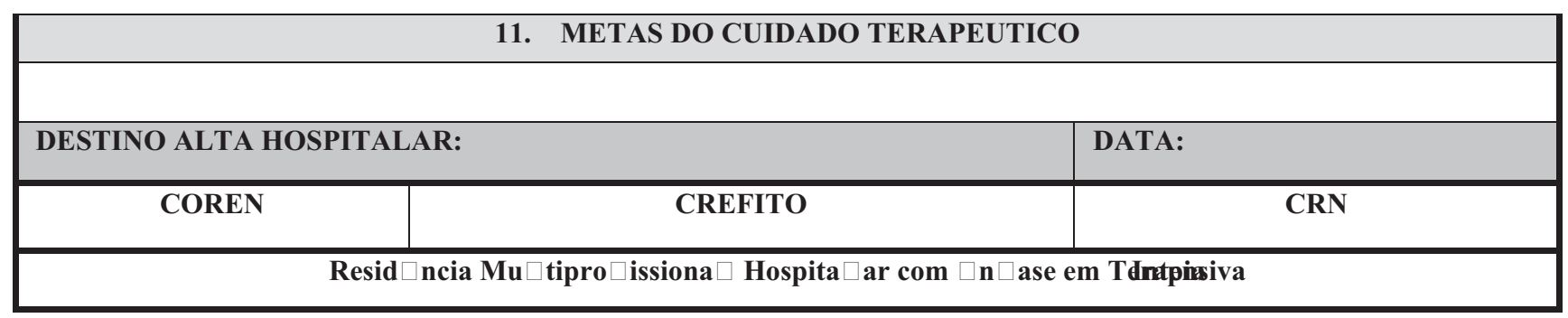

Figura 1 - Frente e verso do instrumento de plano multiprofissional de alta em terapia intensiva. Rio Branco, Acre, 2017.

\section{DISCUSSÃO}

O instrumento foi construído e validado para uso no planejamento multiprofissional de alta da UTI adulto. A produção e avaliação para validação do instrumento de sistematização da continuidade do cuidado, após alta da unidade de terapia intensiva, permite o prosseguimento da atenção à saúde por meio de estratégias de comunicação entre os vários profissionais e a família e incorporação de conhecimentos das múltiplas especialidades, possibilitando a confecção de um roteiro que contenha todas as informações referentes ao paciente, durante seu período de internação, a fim de facilitar a continuidade do processo de cuidado e o alcance de metas previamente estabelecidas ${ }^{8,12}$.

A validação do conteúdo do plano multiprofissional de alta foi de grande importância para o uso e aplicação dentro da realidade das unidades hospitalares ao qual será implantado. O processo de validação permitiu o alinhamento das condutas e do registro de informações relevantes que fizeram parte do processo terapêutico do indivíduo em sua internação e que serão indispensáveis para a sequência de uma assistência de qualidade quando da alta da UTI ${ }^{13}$.

Dispor do instrumento de plano de alta inserido numa unidade hospitalar viabiliza a comunicação e garante o cuidado multiprofissional continuado e integral. ${ }^{14}$ Incorpora, ainda, o familiar/cuidador como ator fundamental do cuidado, a fim de auxiliar o processo de desospitalização de forma rápida e segura, eliminando possíveis efeitos deletérios como tempo prolongado de internação e readmissões evitáveis ${ }^{6}$.

O papel da equipe multiprofissional, nesse processo, é crucial, uma vez que uma alta eficaz deve contar com atuação pluralizada das diferentes áreas envolvidas na assistência, ou seja, não se deve limitar a alta hospitalar ao modelo biomédico, fragmentando o seu atendimento. As demais profissões que atuam na UTI devem fazer parte desse processo, permitindo a intervenção holística na continuidade da assistência ${ }^{15,16}$.

Dentre as profissões, a enfermagem tem participação ativa no gerenciamento durante toda a hospitalização dos pacientes, além de integrar, educar e dinamizar a interlocução das estratégias de intervenções para todos os profissionais e familiares visando a promoção, manutenção, prevenção e recuperação em saúde com o olhar individualizado, ${ }^{6,17}$ sendo contemplado no instrumento as intervenções e prescrições de enfermagem. Um plano de alta realizado pelo enfermeiro proporciona uma forma organizada de desenvolver os cuidados determinados pelas condições específicas de cada paciente ${ }^{18,19}$.

Para o cuidado continuado e integral, a inserção do profissional de fisioterapia é relevante por considerar aspectos específicos relacionados à recuperação pulmonar, ao movimento, funcionalidade e autonomia dos indivíduos, promovendo intervenções direcionadas e progressivas para a independência funcional do paciente ${ }^{20,21}$.

Percebeu-se a necessidade de inclusão da avaliação do risco de broncoaspiração, por ser uma complicação frequente em consequência da diminuição ou ausência dos reflexos de proteção de vias aéreas ${ }^{22,23}$. Também da classificação da força muscular por meio da escala de MRC, em que são estipulados os movimentos que serão avaliados para graduação da presença ou ausência de déficit da força muscular. Sendo ainda incluída a avaliação do tônus muscular que classifica tensão do músculo em repouso, que indica alterações como hipotonia e hipertonia ${ }^{24}$.

O cuidado nutricional é essencial durante todo o período de internação dos pacientes, visto que as implicações da desnutrição sobre a evolução das doenças atuam como fatores coadjuvantes na morbimortalidade ${ }^{25}$, 
sendo assim, uma terapia nutricional adequada deve ser oferecida ao paciente com o objetivo de recuperar e/ou manter o estado nutricional, considerando que a nutrição e alimentação são determinantes e condicionantes da saúde ${ }^{26}$.

O item referente ao estado nutricional foi inserido no instrumento, face à importância da avaliação nutricional utilizada como diretriz para a definição de estratégias alimentares na prevenção e tratamento dos agravos e deficiências nutricionais na hospitalização ${ }^{27}$.

$\mathrm{Na}$ atenção psicossocial, a abordagem da atuação do profissional de psicologia se deve pela relevância da humanização em todo o processo de hospitalização, observando que neste processo há rupturas dos vínculos com amigos, familiares e no cotidiano ocorrendo então a vulnerabilidade, a desordem sentimental e psicológica do paciente como também dos seus amigos e familiares ${ }^{28}$.

Um item específico para orientações gerais do cuidado foi incorporado ao plano de alta, local onde deverão ser registradas metas e enfoques para continuação dos cuidados iniciados no âmbito da UTI. As orientações repassadas durante e ao final do período de internação na unidade favorecem a maior compreensão dos processos de cuidado, diminuindo a ansiedade, a insegurança e os desconhecimentos, além de prevenir condutas inadequadas no manejo do paciente ${ }^{29}$. Tem papel importante, também, na prevenção, promoção e cuidado do indivíduo, otimizando sua qualidade de vida ${ }^{30}$.

Foi solicitada a exclusão dos itens que retratam sobre aspecto, quantidade e cor da secreção traqueal, no entanto, a sua exclusão não foi aceita pelos pesquisadores pela importância dessas informações como método de predição de infecção no trato respiratório, além de sua relevância na manutenção da permeabilidade da via aérea garantido boa ventilação e oxigenação ${ }^{31}$.

A validação deste conteúdo foi essencial para a construção do instrumento, porém apresentou limitações exigindo grande atenção por parte dos pesquisadores para interpretação das informações coletadas e posterior incorporação das sugestões dadas pelos avaliadores nos domínios e itens do instrumento. Houve também dificuldade em conseguir um contingente de profissionais especialistas na área, com disponibilidade para realizar avaliação do instrumento.
Potencialidades foram percebidas durante toda a construção e validação do instrumento de plano multiprofissional de alta. O conhecimento que envolve as etapas para a sua realização, a sensibilização e motivação dos profissionais quanto à importância dessa estruturação, além do fortalecimento do elo entre academia e serviço público de saúde (Rever o texto desse parágrafo). Outro ponto salutar foi a participação de profissionais de diferentes áreas envolvidas no cuidado, além do fato de que sua criação se deu por pesquisadores atuantes diretamente no serviço de terapia intensiva.

\section{CONCLUSÃO}

No presente estudo foi construído e validado o conteúdo de um instrumento de plano de alta multiprofissional para uso em UTI adulto com sucesso, que finalizou com 11 domínios e 55 itens, considerando que cada item de avaliação obteve um grau de concordância que variou entre $85,0 \%$ e 100,0\%. A utilização do referido instrumento possibilitará a sistematização da continuidade do cuidado após alta hospitalar por permitir a comunicação entre os vários profissionais e a incorporação de conhecimentos das múltiplas especialidades. Assim o instrumento é um valioso roteiro de informações dos pacientes críticos visando o seguimento do processo de cuidado para o alcance do estabelecimento do estado de saúde dos mesmos.

No entanto, deve-se destacar que a validação de conteúdo é uma etapa fundamental e inicial do instrumento de plano de alta multiprofissional para uso em UTI adulto, sendo importante sua aplicação em pacientes para realizar o estudo de sua qualidade psicométrica.

Espera-se com este estudo instigar o conhecimento dos profissionais acerca da importância de uma assistência sistematizada, bem como de registros, metas e planejamento para uma alta eficaz, além da sensibilização quanto ao real ganho no uso de instrumentos devidamente construídos, validados e, portanto, com rigor científico em benefício do paciente crítico. 


\section{REFERÊNCIA}

1. Nascimento KC, Gomes AMT, Erdmann AL. A estrutura representacional do cuidado intensivo para profissionais de Unidade de Terapia Intensiva móvel. Rev esc enferm USP. 2013; 47(1):176-84. DOI: 10.1590/ S0080-62342013000100022.

2. Araújo TG, Rieder MM, Kutchak FM, Franco Filho JW. Readmissóes e óbitos após a alta da UTI - um desafio da terapia intensiva. Rev Bras Ter Intensiva. 2013; 25(1):32-8. DOI: 10.1590/S0103507X2013000100007.

3. Silva MCM, Sousa RMC, Padilha KG. Destino do paciente após alta da unidade de terapia intensiva: unidade de internação ou intermediária?. Rev Latino-Am Enfermagem. 2010; 18(2):224-32. DOI: 10.1590/ S0104-11692010000200013.

4. Mendes MMR, Alvarenga MRM. Percepção da Enfermagem sobre reinternações e alta hospitalar de idosos: Resultados preliminares da análise de conteúdo. Rev latino-Am Enfermagem. 2012; 8(2):111-2. DOI: $10.1590 /$ S0104-11692000000200017.

5. Alochio KV, Cruz ICF. Security and preparation of patient on discharge process of UTI: systematic review of literature for a clinical protocol. J Specialized Nursing Care. 2016; 8(1):1-19. Disponível em: http://www.jsncare.uff.br/index.php/ssncare/article/view/2804/679.

6. Delatorre PPG, Sá SPC, Valente, GSC, Silvino ZR. Planning for hospital discharge as a strategy for nursing care: integrative review. Rev Enferm. 2013; 21(2):7151-9.

7. Correia JN, Bonette A. Avaliação do risco de desenvolvimento de lesão tecidual por pressão em clientes internados na unidade de terapia intensiva. Rev Saúde e Pesquisa. 2011; 4(1):123-7. Disponível em: http:// periodicos.unicesumar.edu.br/index.php/saudpesq/ article/view/1597/1212.

8. Alexandre NMC, COLUCI MZO. Validade de conteúdo nos processos de construção e adaptação de instrumentos de medidas. Rev Ciênc. Saúde cole- tiva. 2011; 16(7): 3061-68. DOI: 10.1590/S141381232011000800006 .

9. Mendes KDS, Silveira RCCP, Galvão CM. Revisão integrativa: método de pesquisa para a incorporação de evidências na saúde e na enfermagem. Texto contexto - enferm. 2008; 17(4):758-64. DOI: 10.1590/S010407072008000400018 .

10. Silva AES, Pontes UO, Genzini T, Prado PR, Amaral TLM. Revisão integrativa sobre o papel do enfermeiro no pós-transplante renal. Cogitare Enferm. 2014; 19(3):597-603. Disponível em: http://www.saude. ufpr.br/portal/revistacogitare/wp-content/uploads/sites/28/2016/10/34414-140478-1-PB.pdf.

11. Pasquali L. Instrumentação psicológica: Fundamentos e práticas. Porto Alegre: Artmed; 2010.

12. Mendonça SCB, Zanetti ML, Sawada NO, Barreto IDC, Andrade JS, Otero LM. Construction and validation of the Self-care Assessment Instrument for patients with type 2 diabetes mellitus. Rev Latino-Am Enfermagem. 2017; 25: e2890. DOI: 10.1590/1518-8345.1533.2890.

13. Araújo DS, França AF, Mendonça JKS, Bettencourt ARC, Amaral TLM, Prado PR. Construção e validação de instrumento de sistematização da assistência de enfermagem em terapia intensiva. Rev Rene. 2015; 16(4):4619. DOI: 10.15253/2175-6783.2015000400002.

14. Bôas MLCV, Shimizu HE, Sanches MN. Elaboração de instrumento de classificação de complexidade assistencial de pacientes em atenção domiciliar. Rev Esc Enferm USP. 2016; 50(3):434-41. DOI: 10.1590/ S0080-623420160000400009.

15. Gelbcke FL, Matos E, Sallum NC. Desafios para a integração multiprofissional e interdisciplinar. Rev. Actas de saúde Coletiva. 2012; 6(4):31-9.

16. Garske CCD, Brixner B, Freitas AP, Schneider APH. Avaliação das interações medicamentosas potenciais em prescrições de pacientes em unidade de terapia intensiva. Rev Saúde e Pesquisa. 2016; 9(3):483-90. DOI: 10.17765/1983-1870.2016v9n3p483-490. 
17. Oliveira FF. Educação em saúde no contexto da alta hospitalar de paciente de unidade de terapia intensiva. Revista Multitexto. 2016; 4(1):38-43. Disponível em: http://www.ead.unimontes.br/multitexto/index. php/rmcead/article/view/172.

18. Cruz IML, Mantovani MF. Orientação de enfermagem para a alta hospitalar do paciente neoplásico. Rev Cogitare enfermagem. 2014; 19(4):687-93. DOI: 10.5380/ce.v19i4.36261.

19. Marinho PML, Campos MPA, Rodrigues EOL, Gois CFL, Barreto IDC. Construção e validação de instrumento de Avaliação do Uso de Tecnologias Leves em Unidades de Terapia Intensiva. Rev Latino-Am Enfermagem. 2016; 24: e2816. DOI: 10.1590/1518-8345.1002.2816.

20. Lunardi AC, Resende JM, Cerri OM, Carvalho CRF. Efeito da continuidade da fisioterapia respiratória até a alta hospitalar na incidência de complicações pulmonares após esofagectomia por câncer. Rev Fisioter Pesqui. 2008; 15(1):72-5. DOI: 10.1590/\$180929502008000100012.

21.Dietrich, C.; Cardoso, J.R.; Vargas, F.; Sanchez, E.C.; Dutra, F.H.; Moreira, C, et al. Capacidade funcional em idosos e idosos mais velhos após alta da unidade de terapia intensiva. Coorte prospectiva. Rev Bras Ter Intensiva. 2017; 29(3):293-302. DOI: 10.5935/0103$507 x .20170055$.

22. Leal AA. A Iatrogenia na enfermagem. Rev. Eletrônica da Univa. 2013; 01(9):102-8.

23. Bispo MM, Dantas ALM, Silva PKA, Fernandes MICD, Tinôco JDS, Lira ALBC. Diagnóstico de enfermagem risco de aspiração em pacientes críticos. Esc. Anna Nery. 2016; 20(2):357-62. DOI: 10.5935/14148145.20160049.

24. Mussalem MAM, Silva ACSV, Couto LCLV, Marinho L, Florencio ASM, Araújo VS, et al. Influência da mobilização precoce na força muscular periférica em pacientes na Unidade Coronariana. ASSOBRAFIR Ciência. 2014; 5(1): 77-88. Disponivel em: http://www.uel.br/revistas/uel/index.php/rebrafis/article/view/17530/14460
25. Silva MTG, Oliveira MM. A importância da terapia nutricional nas Unidades de Terapia Intensiva. BRASPEN. 2016; 31(4):347-56. Disponivel em: http://www.braspen.com.br/wp-content/uploads/2017/02/12-AO-A-import\% $\mathrm{C} 3 \% \mathrm{~A} 2$ ncia-da-terapia-nutri.pdf.

26. Brasil. Lei $n^{\circ} 8.080$, de 19 de Setembro de 1990. Condições para a promoção, proteção e recuperação da saúde, a organização e o funcionamento dos serviços correspondentes e dá outras providências. Diário Oficial da União 20 de Set 1990; Seção 1: 18055.

27. Duchini L, Jordão AA, Brito TT, Diez-Garcia RW. Avaliação e monitoramento do estado nutricional de pacientes hospitalizados: uma proposta apoiada na opinião da comunidade científica. Rev Nutri. 2010; 23(4):51322. DOI: $10.1590 / S 1415-52732010000400002$.

28. Camponagara S, Viero CM, Pinno C, Soares SGA, Rodrigues IL, Cielo C. Percepções de pacientes pós-alta da unidade de cuidados intensivos sobre a hospitalização nesse setor. R. Enferm. Cent. O. Min. 2015 jan/ abr; 5(1):1505-1513. DOI:10.19175

29. Pompeo DA, Pinto MH, Cesarino CB, Araújo RRDF, Poletti NAA. Atuação do enfermeiro na alta hospitalar: reflexões a partir dos relatos de pacientes. Acta paul enferm. 2007; 20(3):345-50. DOI: 10.1590/S010321002007000300017.

30. Mondadori AG, Zeni EM, Oliveira A, Silva CC, Wolf VLW, Taglietti M. Humanização da fisioterapia em Unidade de Terapia Intensiva Adulto: estudo transversal. Fisioter Pesqui. 2016; 23(3):294-300. DOI: $10.1590 / 1809-2950 / 16003123032016$.

31. Ranzani OT, Forte DN, Forte AC, Mimica I, Forte WCN. Utilidade da avaliação de bactérias revestidas por anticorpos em aspirados traqueais para o diagnóstico de pneumonia associada à ventilação mecânica: um estudo caso-controle. J bras pneumol. 2016; 42(3):20310. DOI: $10.1590 / \mathrm{S} 1806-37562015000000244$.

Recebido em: 08/11/2017 Aceito em: 22/02/2018 